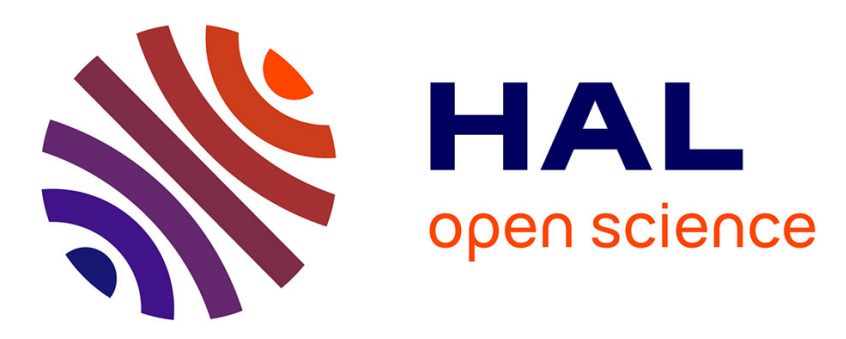

\title{
Experimental validation of in-hand planar orientation and translation in microscale.
}

Benoît Brazey, Redwan Dahmouche, Jean-Antoine Seon, Michaël Gauthier

\section{To cite this version:}

Benoît Brazey, Redwan Dahmouche, Jean-Antoine Seon, Michaël Gauthier. Experimental validation of in-hand planar orientation and translation in microscale.. Intelligent Service Robotics, 2016, Multiscale Manipulation Toward Robotic Manufacturing Technologies., 9 (2), pp.101-112. hal-01303485

\section{HAL Id: hal-01303485 \\ https://hal.science/hal-01303485}

Submitted on 18 Apr 2016

HAL is a multi-disciplinary open access archive for the deposit and dissemination of scientific research documents, whether they are published or not. The documents may come from teaching and research institutions in France or abroad, or from public or private research centers.
L'archive ouverte pluridisciplinaire HAL, est destinée au dépôt et à la diffusion de documents scientifiques de niveau recherche, publiés ou non, émanant des établissements d'enseignement et de recherche français ou étrangers, des laboratoires publics ou privés. 


\title{
Experimental Validation of In-Hand Planar Orientation and Translation in Microscale
}

\author{
B. Brazey, R. Dahmouche, J.A. Seon \& M. Gauthier
}

\begin{abstract}
This paper presents the experimental validation of automatic dexterous in-hand manipulation of micro-objects. Currently, precise handling of micro-objects is still a challenge especially when large rotations are required. Indeed, the current dexterity of microgrippers is still very low and only some small range rotations have been shown. Although, the robotic hands in the macroscale have better capabilities, they are not able to manipulate micro-objects. The proposed approach extends the capabilities of dexterous macrohands to the microgrippers enabling dexterous micro-manipulation. Design rules of the microhand fingers and trajectories enabling micro-manipulation are proposed. The developed methods are validated by simulation and on an original experimental prototype having three fingers ( $7 \mu \mathrm{m}$ in diameter). Half turns of $220 \mu \mathrm{m}$ square objects demonstrates the relevance of the approach which opens the way to new advanced in-hand micro-manipulation and micro-assembly methods.
\end{abstract}

\section{INTRODUCTION}

The development of microtechnologies in fields such as biomedical instrumentation, watch industry and optical microsystems, in which several technologies has to be merged in a unique system, has increased the applicative interest of micro-assembly in micromanufacturing. However, most of micro-assembly operations are still done manually because of the lack of automated systems able to perform complex operations especially when orientation positioning is critical.

The first challenge for micro-assembly mentioned in a large majority of papers in the literature deals with the adhesion forces (mainly van der Waals, electrostatic and capillary forces) which are larger than volume forces (weight and inertia). The manipulation strategies in microscale have to take this constraint into account.

The development of microassembly methods is also limited by the lack of precision of multi-axes rotation systems which are currently not able to provide sufficient positioning accuracy for precise micro-assembly [1], [2]. The classical structure of an industrial robot consisting in bodies, joints and actuators linked in serial or parallel architecture are not efficient in microscale. Indeed, the eccentricity error of a conventional joint (e.g. several microns) becomes comparable to the request manipulation precision when manipulating a micro-object. The use of a serial robotic structure composed of several rotational stages is only possible for large microobject having a size down to $100 \mu m$ [3]. Moreover the rotation of a whole robot to manipulate only microparts significantly increases the moved mass and thus reduces the

All authors are with FEMTO-ST Institute, AS2M department, Univ. Bourgogne Franche-Comté, Univ. de Franche-Comté/CNRS/ENSMM, 24 rue Savary, F-25000 Besançon, France. \{benoit.brazey, redwan.dahmouche, ja.seon, michael.gauthier\}@femto-st.fr potential throughput. The last drawback is the high volume needed to achieve the rotations which limits the application of this solution especially in confined environments (Scanning Electron Microscope, for instance). To work around this problem, self-assembly techniques have been developed [1], [4]. However, these techniques have limited application cases.

Increasing the dexterity of the microgrippers is one of the most studied solutions to overcome the precision lack in rotation. Indeed, in-hand manipulation allows to perform local rotations without using conventional rotation stages. Previous works have demonstrated the feasibility of automatic and semi-automatic handling using two [5]-[8], three [9] or four [10] probes having several degrees of freedom (DoF). However, the manipulated objects were limited to microspheres except in [9] where more complex objects have been manipulated using three probes having 6 DoF (two mobile fingers and a static one). In [11] an integrated dexterous gripper with 2 fingers having 3 DoF each was also used for micro-parts inspection. In all these works, no regrasping strategy was considered during the manipulation and the contact points between the gripper and the manipulated object were kept as stable as possible which limits the manipulation to small rotation adjustments. In [12]-[14], large object rotations have been performed thanks to two compliant microtweezers. However, the manipulation process requires placing the manipulated object on a specially designed shape for object regrasp which limits the versatility of the approach. To enhance the dexterity in micro-manipulation, multiple fingers, having multiple DoF, are needed to enable online regrasping.

In this paper, we propose a new approach of automatic multi-fingers dexterous micro-manipulation using rolling contacts and finger gaiting strategies. Based on our knowledge, this work represents the first in-hand manipulation performed in microscale, which allows $180^{\circ}$ positioning range. The experimental validation of the concept is performed on several micro-objects of different sizes (from $120 \mu \mathrm{m}$ to $400 \mu \mathrm{m})$.

The next section describes the proposed manipulation strategies and formalizes the dexterous micro-manipulation problem. Section III details the finger gaiting trajectory generation and the kinematics behind it. Finally, section IV describes the experimental setup and shows the obtained results in micro-objects reorientation, followed by a conclusion and perspectives. 


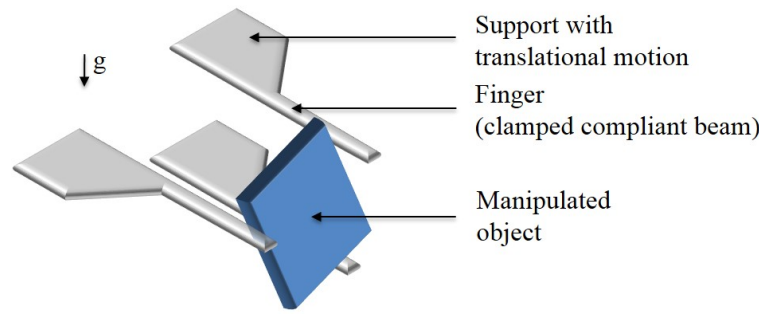

Fig. 1: Proposed in-hand manipulation principle : the manipulated object is rotated using 3 microfingers moving in a plane. Each finger has a 2DoF translation motion.

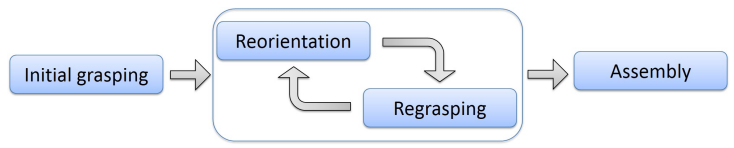

Fig. 2: The main dexterous manipulation steps. After the initial grasping, the manipulated object is reoriented using two fingers until the stability limit is reached, whereupon the object regrasp using the third finger is performed. The reorientation and regraping operations are repeated until the desired orientation is reached. Finally, the object is released, inserted or assembled.

\section{HANDLING PRINCIPLE AND PROBLEM DEFINITION}

\section{A. MANIPULATION APPROACH}

The approach of the proposed dexterous manipulation method is to translate, in the plane ( $x$ and $y$ axes), $\mathrm{N}$ compliant beams which will act as fingers to handle microobjects (as depicted in Fig.1). The fingers will apply a bending force on the manipulated object to move it along a reference trajectory. To perform pick-and-place operations using dexterous micro-manipulation approach, the handling sequence is decomposed into four major steps (see Fig.2):

1) Grasping the object from the substrate

2) Translation of the fingers and in-hand rotation of the object

3) Finger gaiting and in-hand regrasping of the object (and eventually going to step 2)

4) Release or assembly of the object

Each step will imply several constraints to take into account in order to perform a stable handling. In absence of force sensors able to measure grasping force in microscale, fingers compliance will be exploited to control the grasping forces. The next subsection focuses on the three first steps and presents their characteristics.

\section{B. NOTATIONS AND HYPOTHESES}

The notations of this section are illustrated in Fig.3. In accordance with our experimental device, we focus on the case where cylindrical fingers are used to manipulate an object with opposite edges. We assume that all the fingers have identical shapes and mechanical behaviors. The main parameters and their definition are summarized in Table I.
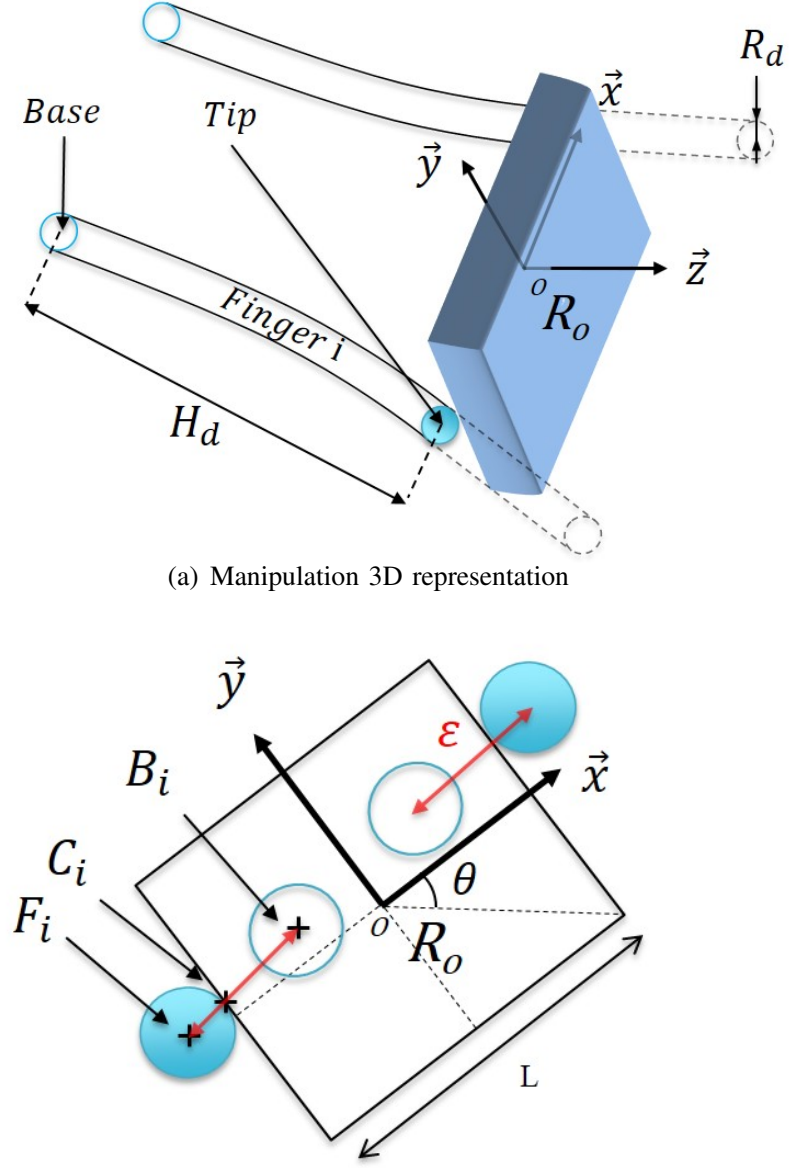

(b) Manipulation 2D representation (projection on $(O x y)$ )

Fig. 3: The proposed dexterous micro-manipulation solution (a) illustrated in 3D and (b) its 2D projection. Compliant beams are used as fingers to grasp and manipulate the object by rolling without sliding. The desired forces are obtained by controlling the fingers bending thanks to the fingers bases positions calculated as a function of the contact points distance.

\section{Frames and points:}

We consider compliant fingers whose base is translated along $2 \mathrm{DoF}$, and which are in contact with a square shaped object. $L$ defines the length of the edge of the object. The origin of the object frame $\mathcal{R}_{0}=\{\mathrm{O},(\mathrm{x}, \mathrm{y}, \mathrm{z})\}$ is chosen as the center of the closest object surface to the actuation as depicted in Fig.3.a. The $x \& y$ axes are parallel to the object edges.

$B_{i}=\left(\begin{array}{l}x_{b_{i}} \\ y_{b_{i}}\end{array}\right)$ is the projection of the $i^{t h}$ finger base center on the (Oxy) plan.

$C_{i}=\left(\begin{array}{l}x_{c_{i}} \\ y_{c_{i}}\end{array}\right)_{\mathcal{R}_{o}}$ is the contact point between the $i^{\text {th }}$ finger and the object on the (Oxy) plan.

$F_{i}$ is the intersection between the $i^{\text {th }}$ finger central axis and the $(\mathrm{O} x y)$ plan. 


\begin{tabular}{|c|c|}
\hline Notation & Parameter \\
\hline$x_{b_{i}}, y_{b_{i}}$ & $i^{\text {th }}$ finger base position \\
$x_{c_{i}}, y_{c_{i}}$ & $i^{\text {th }}$ finger contact position \\
${ }^{j} x_{c_{i}},{ }^{j}, y_{c_{i}}$ & $i^{\text {th }}$ finger initial contact position \\
& (before the $j^{t h}$ rotation) \\
$L$ & Object length \\
$\mathrm{h}$ & Object depth \\
$\Delta \theta$ & Object orientation variation \\
$R_{d}$ & Finger radius \\
$H_{d}$ & Finger length \\
$\varepsilon, \varepsilon_{x_{i}}, \varepsilon_{y_{i}}$ & $i^{\text {th }}$ finger bending \\
$F_{x}^{i / 0}, F_{y}^{i / 0}$ & Finger yield \\
$R_{e}$ & Finger Young Modulus \\
$E$ & Finger flexural stiffness \\
$\mathrm{k}$ & $i^{\text {th }}$ finger applied force on the object \\
$F_{a d}^{s / o}$ & Pull-off force between the object and the substrate \\
$f_{a d}^{s / o}$ & Pull-off surface force between the object and the substrate \\
$S_{\text {contact }}$ & Contact surface between the object and the substrate \\
$\mu$ & Friction coefficient between the object and a finger \\
\hline
\end{tabular}

TABLE I: Main parameters used in the problem formulation.

\section{Geometrical and material properties:}

The parameters describing the end-effectors (fingers) are: the radius $R_{d}$, the flexure stiffness $k$ (defined by the EulerBernoulli formula), the Young Modulus $E$ and the mechanical yield $R_{e}$. The contact between the object and a finger is considered as a punctual contact, with a friction coefficient $\mu . H_{d}$ defines the distance between the contact point and the finger base.

\section{Grasping force control:}

A contact between the $i^{\text {th }}$ finger and the object induces a bending $\overrightarrow{F_{i} B_{i}}$ which is proportional to the applied force on the object $\overrightarrow{R_{\text {ext }}^{i / o}}$ :

$$
\overrightarrow{R_{e x t}^{i / o}}=\left(\begin{array}{c}
F_{x}^{i / o} \\
F_{y}^{i / o}
\end{array}\right)=k \overrightarrow{F_{i} B_{i}}=k\left(\begin{array}{c}
\varepsilon_{x_{i}} \\
\varepsilon_{y_{i}}
\end{array}\right) .
$$

The bending $\overrightarrow{F_{i} B_{i}}$ is the distance from the tip to the base of the finger in projection in the (Oxy) plan (see Fig.3.b). $\varepsilon$ defines the bending amplitude: $\varepsilon=\sqrt{\varepsilon_{x}^{2}+\varepsilon_{y}^{2}}$.

As we consider in the study that the contacts are on parallel edges, $F_{x}^{i / o}$ and $F_{y}^{i / o}$ will always respectively represent the tangential and normal component of applied forces. Knowing the fingers stiffness, the forces applied on the object can be controlled through the fingers bending thanks to the fingers bases positions which can be calculated as a function of the contact points distance. The case in which the fingers are in contact with the edges parallel to $\vec{x}$ is not detailed in this paper but is similar to the previous case.

\section{The influence of the adhesive frictional contacts:}

In microscale, volume forces such as weight can be considered negligible compared to the blocking forces applied by the fingers. Adhesion forces (van der Waals, electrostatic, capillarity...) will then have a significant influence on the manipulation and have to be considered. As the manipulation strategy implies rolling without sliding, the finger trajectory will ensure that no slippage will occur during the manipulation. This means that the sliding limit defined by a friction cone will not be exceeded. Let $F_{a d}^{s / o}$ be the adhesion force amplitude between the substrate and the considered object (pull-off force) and $F_{a d}^{f / o}$ the adhesion force amplitude between a finger and the object.

The adhesion will increase the contact force between a finger and the object, and between the object and the substrate. In other words, adhesion improves the stability during the in-hand manipulation but induces difficulties during the pick-up from the substrate. Nevertheless, adhesion amplitude is highly variable and unpredictable due to its dependence on the characteristics of the contact surface between the considered objects (tribology of surfaces, applied forces, etc.). This stochastic behavior will imply different hypotheses on adhesion during the four main manipulation steps considering always the worst case.

\section{MANIPULATION STABILITY}

The effect of adhesion forces in microscale requires particular care to guarantee stable in-hand micro-manipulation process. We propose to study the stability of the steps required for successful manipulation: grasping, reorientation and regrasping.

\section{Initial grasping and pull-off:}

We assume that the parts to handle are initially laid on a planar surface (the substrate). For the successful pick-up of the object using two fingers (Fig.4), it is necessary to overcome pull-off force $F_{a d}^{s / o}$ between the object and the substrate (plan/plan contact). During this step, the critical case corresponds to the maximal possible value of the pulloff force $F_{a d}^{s / o}$, and the minimal adhesion force between the fingers and the object. We consequently assume that there is no adhesion between the fingers and the object. Considering that the two fingers have a friction coefficient $\mu$, to pick-up the object, the normal force to apply by each finger is:

$$
\left\|\overrightarrow{F_{x}^{1 / o}}\right\|=\left\|\vec{F}_{x}^{2 / o}\right\| \geq \frac{1}{2 \mu} \cdot F_{a d}^{s / o}
$$

The minimum admissible grasping force applied by each finger is then given by:

$$
F_{\text {grasping }}=\left(\left\|\overrightarrow{F_{x}^{1 / o}}\right\|^{2}+\left\|\overrightarrow{F_{y}^{1 / o}}\right\|^{2}\right)^{\frac{1}{2}} \geq \frac{1}{2}\left(1+\frac{1}{\mu^{2}}\right)^{\frac{1}{2}} F_{a d}^{s / o}
$$

\section{Object reorientation:}

During the in-hand reorientation, the displacement of the contact points on the object and the evolution of the forces directions applied by the pair of fingers modifies the orientation equilibrium of the object which makes it 


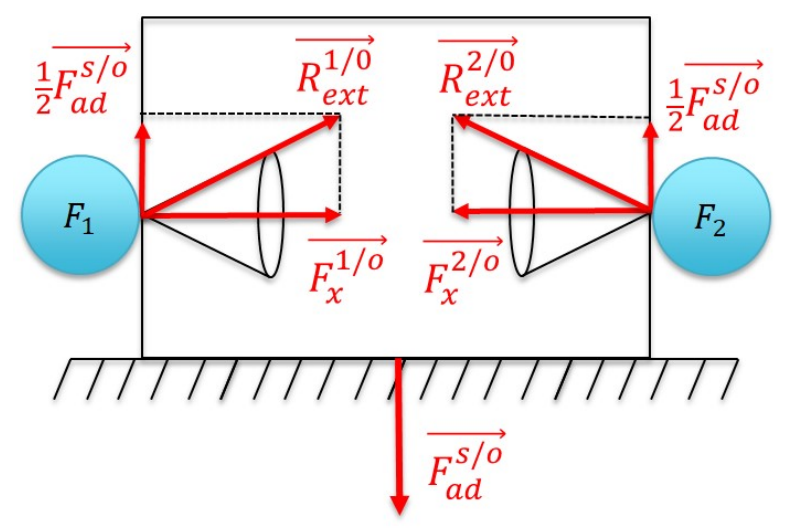

Fig. 4: Representation of the grasping forces exerced by 2 fingers to detach an object from the substrate. The condition to achieve the object pick-up is that the exerted grasp forces overcome the pull-off force.

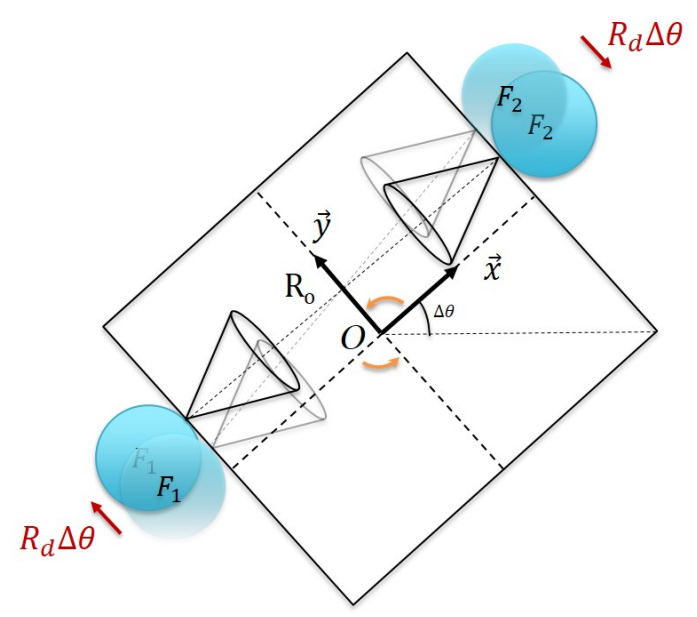

Fig. 5: Finger rolling while handling an object: rolling induces a contact point variation that must be considered to ensure a stable grasp.

rotate as shown in Fig.5. The most critical situation which is considered during this step is when the adhesion forces that contribute to stabilise the grasp are null. In absence of external forces (the gravitation force being neglected), the grasp stability while rotating the object is ensured when the applied forces remain in the friction cone. Let us consider the Newton's law applied at the center of the object:

$$
\left(\begin{array}{c}
F_{x}^{1 / o}+F_{x}^{2 / o} \\
F_{y}^{1 / o}+F_{y}^{2 / o} \\
\frac{L}{2}\left(F_{y}^{2 / o}-F_{y}^{1 / o}\right)-F_{x}^{2 / o} y_{c_{2}}-F_{x}^{1 / o} y_{c_{1}}
\end{array}\right)_{\mathcal{R}_{0}}=\overrightarrow{0},
$$

As only two forces are applied on the object respectively in $C_{1}$ and $C_{2}$, we can show that both forces are parallel to $\overrightarrow{C_{1} C_{2}}$. Consequently, the ratio between the tangential and normal forces depends on the contact points positions. Thus, the grasp stays stable as long as:

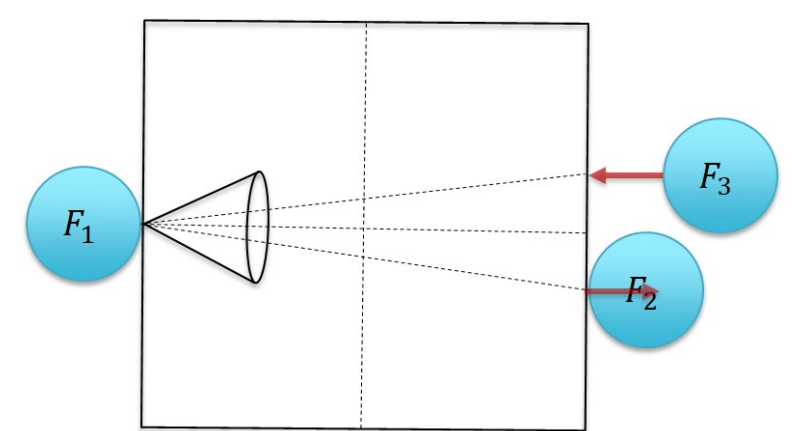

Fig. 6: Example of regrasping using 3 fingers. Geometry of the different elements should enable both initial and final configuration to be stable by applying forces remaining in the friction cone.

$$
\left|\frac{y_{c_{2}}-y_{c_{1}}}{L}\right| \leq \mu
$$

Considering that the fingers can roll on the object without sliding during the reorientation, equation 5 can be expressed as a function of the initial contact points positions ${ }^{1} y_{c_{i}}$ and the object rotation amplitude $\Delta \theta$ as follows:

$$
\left|\frac{{ }^{1} y_{c_{2}}-{ }^{1} y_{c_{1}}-2 R_{d} \Delta \theta}{L}\right| \leq \mu
$$

Let us consider an initial grasping in which contact points $C_{1}$ and $C_{2}$ are in front of each other $\left(\overrightarrow{C_{1} C_{2}}\right.$ is the normal of the grasped object edges). The maximal achievable angle $\Delta \theta$ without regrasping is:

$$
|\Delta \theta| \leq \frac{\mu L}{2 R_{d}}
$$

\section{Regrasping:}

If the required rotation angle is larger than the achievable one using two fingers, the object regrasping using a third finger is needed to continue the rotation (see Fig.6).

The regrasping can be performed if both finger $1 \& 2$ and finger $1 \& 3$ represent stable grasps. Considering both geometrical conditions (5) for each grasp and the fact that the minimal distance between finger centers is $2 R_{d}$, the finger gaiting can be done only if:

$$
R_{d} \leq L \mu
$$

This inequality shows that the regrasping feasibility does not depend on rotation amplitude but only on the relative dimensions between the end-effectors and the object. During the transition between the two configurations, the pull-off forces between the removed fingers and the object may also disturb the grasp. A detailed study of the impact of pull-off force on finger gaiting is presented in [15].

To conclude this section, adhesion is a phenomenom that contributes to stabilize the grasp during in-hand rotation but disturbs pick-up and regrasping operations. Consequently, hypotheses about adhesion are: 
- Pull-off $F_{a d}^{s / o}$ is maximal and adhesion between the object and the fingers $F_{a d}^{f / o}$ is zero during the pick-up. - $F_{a d}^{f / o}$ is minimal for fingers grasping the object and maximal for the finger to detach.

This microscale particularity were considered for the design of the finger. The equations (2) to (8) define a compromise to reach regarding the geometry of the fingers. Indeed, a tradeoff has to be found between both conditions. This point will be tackled in section IV.B.

\section{MOTION PLANNING}

In this section, we are going to propose a formulation of finger trajectories enabling the different manipulation steps.

\section{Initial grasping:}

During the intial grasping step, the fingers are translated from their initial position to the pick-up grasping configuration where two opposite fingers are used (Fig.7.a). Knowing the stiffness of the fingers, the fingers bases positions are calculated to generate the required blocking force $F_{\text {grasping }}$ allowing to overcome the pull-off force as defined in (3). After reaching the required positions, the object is moved up to a position above the substrate.

\section{Rotation:}

Once the object is detached from the substrate, a first rotation is performed by the two fingers used during the pickup step. The fingers trajectories are generated to enable the rolling of the fingers on the object sides. In the case presented here, the center of rotation is chosen as the center of mass of the object as shown in Fig.5. For a given initial contact points ${ }^{1} y_{c_{1}}$ and ${ }^{1} y_{c_{2}}$, the fingers base positions are defined by:

$$
\begin{aligned}
& \left(\begin{array}{l}
x_{b_{1}} \\
y_{b_{1}}
\end{array}\right)_{\mathcal{R}_{0}}=\overrightarrow{O B_{1}}=\left(\begin{array}{c}
-R_{d}-\frac{L}{2}+\varepsilon_{x_{1}} \\
1_{c_{1}}+R_{d} \Delta \theta+\varepsilon_{y_{1}}
\end{array}\right) \\
& \left(\begin{array}{l}
x_{b_{2}} \\
y_{b_{2}}
\end{array}\right)_{\mathcal{R}_{0}}=\overrightarrow{O B_{2}}=\left(\begin{array}{c}
R_{d}+\frac{L}{2}-\varepsilon_{x_{2}} \\
1 y_{c_{2}}-R_{d} \Delta \theta-\varepsilon_{y_{2}}
\end{array}\right)
\end{aligned}
$$

The symmetry of the forces induces a symmetry of the bending: $\varepsilon=\sqrt{\varepsilon_{x_{1}}^{2}+\varepsilon_{y_{1}}^{2}}=\sqrt{\varepsilon_{x_{2}}^{2}+\varepsilon_{y_{2}}^{2}}$. The positions of the fingers bases expressed in the object frame $\mathcal{R}_{0}$ are thus given by:

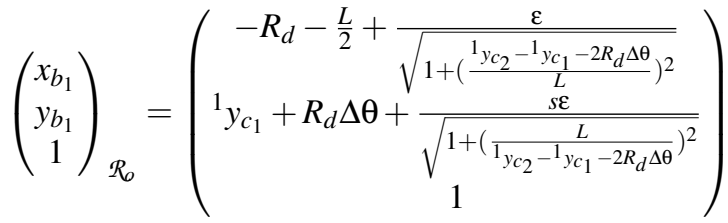

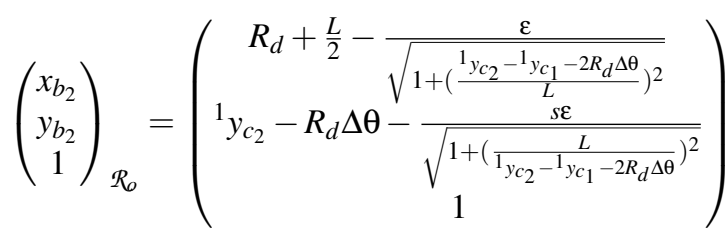

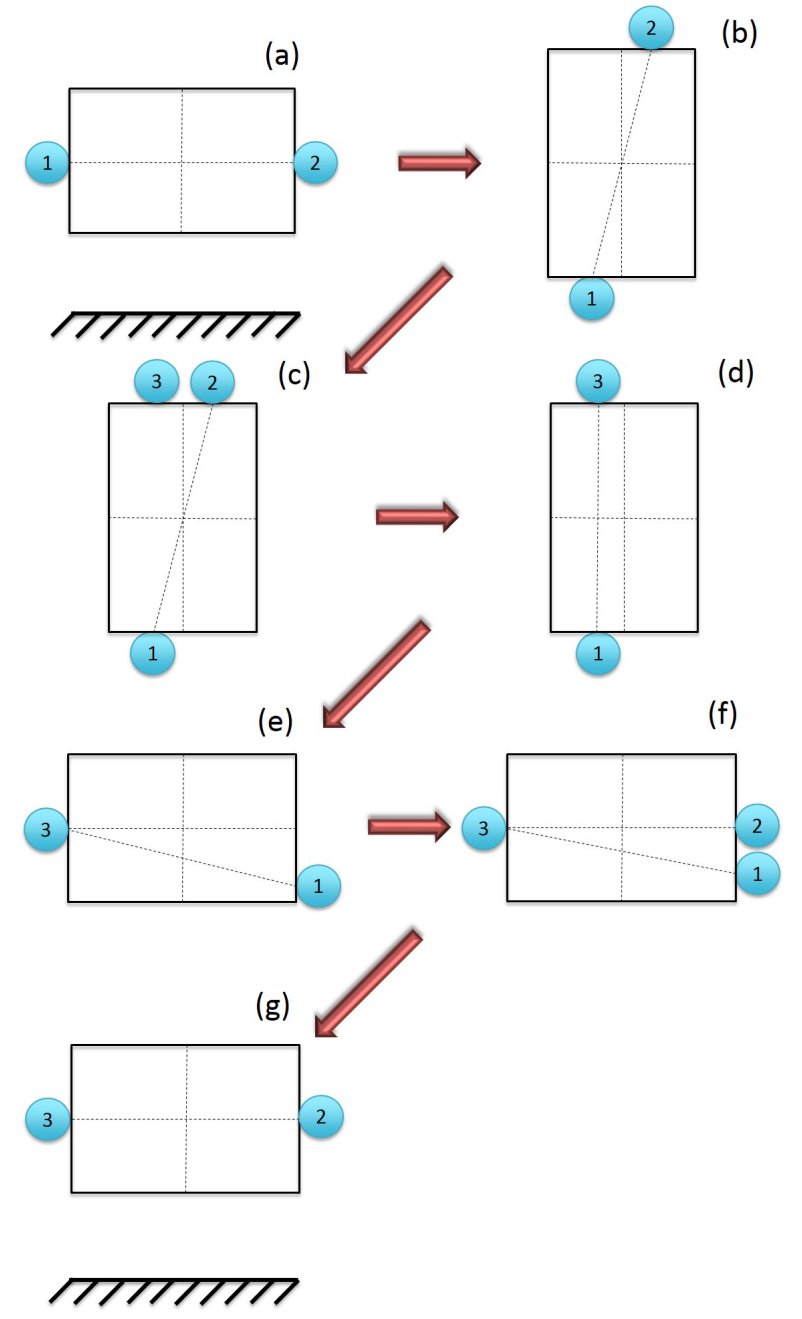

Fig. 7: Dexterous manipulation using rolling without sliding and finger gaiting techniques: the $180^{\circ}$ rotation of the object is obtained by performing a succession of rotations $(a \rightarrow b$, $\mathrm{d} \rightarrow \mathrm{e})$ and regraspings $(\mathrm{b} \rightarrow \mathrm{d}, \mathrm{e} \rightarrow \mathrm{g})$.

, where $s=\operatorname{sign}\left(y_{c_{2}}-y_{c_{1}}\right)$.

These equations describe the base centers $B_{1}$ and $B_{2}$ coordinates expressed in the object frame $\mathcal{R}_{0}$. The fingers trajectories can be expressed in the working frame by simply applying the transformation matrix between both frames:

$$
{ }^{w} X=T^{o} X
$$

where ${ }^{w} X$ and ${ }^{o} X$ are respectively the homogeneous positions of the fingers in the work frame and the object frame and $T$ the transformation matrix between both frames depending on the object pose.

\section{Regrasping:}

When the contact point positions between the fingers and the manipulated object reaches the stability limit or one of the object corners, a regrasping is needed (Fig.7.b $\rightarrow$ Fig.7.d).

The new grasp will be stable if and only if the applied forces are in the friction cone. Finger 3 can then be placed 
on the object respecting the stability condition. The direction of rotation and the fingers accessible workspace will impose the side on which the finger will be placed on (Finger's 1 or Finger's 2 side). The approach trajectory of Finger 3 satisfying the stability condition and the application of desired force is given by:

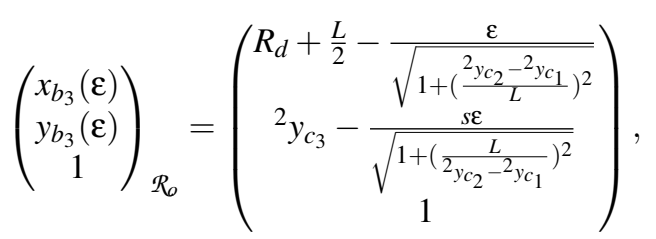

where ${ }^{2} y_{c_{1}}$ and ${ }^{2} y_{c_{3}}$ are respectively the initial positions of Finger 1 and Finger 3, before detaching Finger 2. At last, Finger 2 is detached from the object and the object rotation is resumed.

The two previous sections provided us all the mathematical formulations needed to generate stable trajectories during the three manipulation steps studied. The criteria (3), (7) and (8) respectively define the stability limit of the grasping, in-hand rotation and finger gaiting, while the equations (10), (11) and (12) enable to generate the trajectories.

\section{SIMULATION AND EXPERIMENTAL RESULTS}

\section{A. EXPERIMENTAL SETUP}

The role of the 6-DoF dexterous manipulation system, shown on Fig.8, is to demonstrate the experimental viability of our approach. The experimental system is composed of:

- The actuation system:

6 Smaract SLC-1730 piezoelectric actuators controlled in closed-loop. Travel: $18 \mathrm{~mm}$, repeatability: $1.5 \mu \mathrm{m}$.

- The fingers:

3 carbon fibers (Fig.8 \& Fig.10). Diameter: $7 \mu \mathrm{m}$, approximate length: $350 \mu \mathrm{m}$ (more details on the fibers will be given in the next section).

- The vision system:

Two 1024 x 768 pixels cameras with CCD sensors, a front camera and a side one placed at $45^{\circ}$, both equipped with a $x 5$ zoom.

- Initial positioning of the fingers:

Three Newport M-DS40-XY manual linear stages with $1 \mu \mathrm{m}$ sensitivity.

The architecture of the whole system is presented in Fig.9.

The fingers are carbon microfiber beams which can be translated independently of each other in the plane (Oxy), thanks to the stick-slip actuators. We ensure beforehand that all fingers bases have the same depth by adjusting their position along the $z$ axis using the manual linear stages.

The crystallographic quality of the fibers enables large elastic deformations. The fibers are fixed on the tip of a silicon triangle, as shown in Fig.10. This part has been manufactured from a silicon wafer with a diamond saw and glued on a polymer support. The geometry of the silicon part has been designed to prevent collision during $180^{\circ}$ rotation of the object.

\section{B. DESIGN OF THE FINGERS}

Objects from 100 to $500 \mu \mathrm{m}$ in length \& width and about $100 \mu \mathrm{m}$ in thickness have been considered. The fingers have to be dimensioned in order to satisfy stability criteria enounced in section II, for all considered object sizes. They also have to be rigid enough to reach a sufficient force able to overcome the pull-off forces and sufficiently compliant to allow the control of the forces exerted on the manipulated object. Thus, their dimensions have to allow to:

- Overcome the pull-off force between the object and the substrate during the initial grasping:

To avoid breaking the fingers by applying an excessive force and to prevent collision between the fingers supports (bending higher than the half of the object edge length), we consider the analytical formulation of strain and bending while applying a force on the fingers:

$$
\left\{\begin{array}{l}
\sigma\left(F_{\text {grasping }}\right) \leq R_{e} \\
\varepsilon\left(F_{\text {grasping }}\right) \leq \frac{L}{2},
\end{array}\right.
$$

where $\sigma$ is the internal strain induced by the bending $\varepsilon$ of the carbon fibers. We consider that the maximum pull-off force is proportional to the surface force $f_{a d}^{s / o}$ and the contact surface of the widest manipulated object $S_{\text {contact }}$ :

$$
F_{a d}^{s / o}=f_{a d}^{s / o} S_{\text {contact }}
$$

This equation enables to define the theoretical maximum pull-off force value. The minimum blocking force applied by fingers to ensure a stable initial grasp is calculated from (3):

$$
F_{\text {grasping }} \geq \frac{1}{2}\left(1+\frac{1}{\mu^{2}}\right)^{\frac{1}{2}} f_{\text {ad }}^{s / o} \cdot S_{\text {contact }}
$$

The maximum finger lengths are calculated from the bending and strain formulas for an Euler-Bernoulli beam:

$$
\left\{\begin{array}{r}
H_{d} \leq\left(\frac{3 E \pi R_{d}^{4} L}{4 F_{\text {grasping }}}\right)^{\frac{1}{3}} \\
H_{d} \leq \frac{\pi R_{e} R_{d}^{3}}{2 F_{\text {grasping }}}
\end{array}\right.
$$

- Perform rotations with the highest possible amplitude, without sliding:

As defined in (Fig.7), the amplitude of the rotation $\Delta \theta$ is a function of the end-effector radius $R_{d}$. Moreover, collision due to fingers supports will also limit the rotation amplitude to approximatively $\frac{\pi}{2}$. We choose to maximize the available rotation and to define a $R_{d}$ enabling to perform the maximal rotation $\frac{\pi}{2}$. From (7), $R_{d}$ verifies:

$$
R_{d} \leq \frac{\mu L_{\min }}{\pi}
$$

where $L_{\min }$ is the minimum length of manipulated objects. 


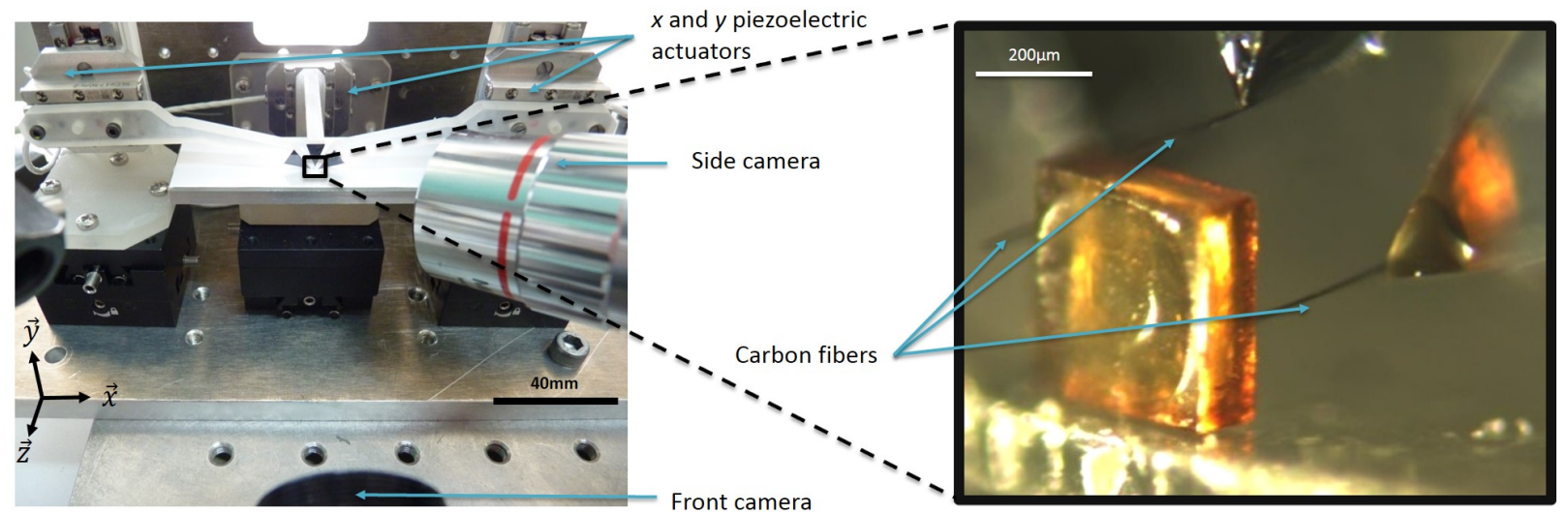

Fig. 8: The dexterous micro-manipulation station. 3 cylindrical fingers are mounted on a support and translated by the actuation system to perform manipulations. Two cameras allow to calibrate and visualise the 6 DoF micro-manipulation system.

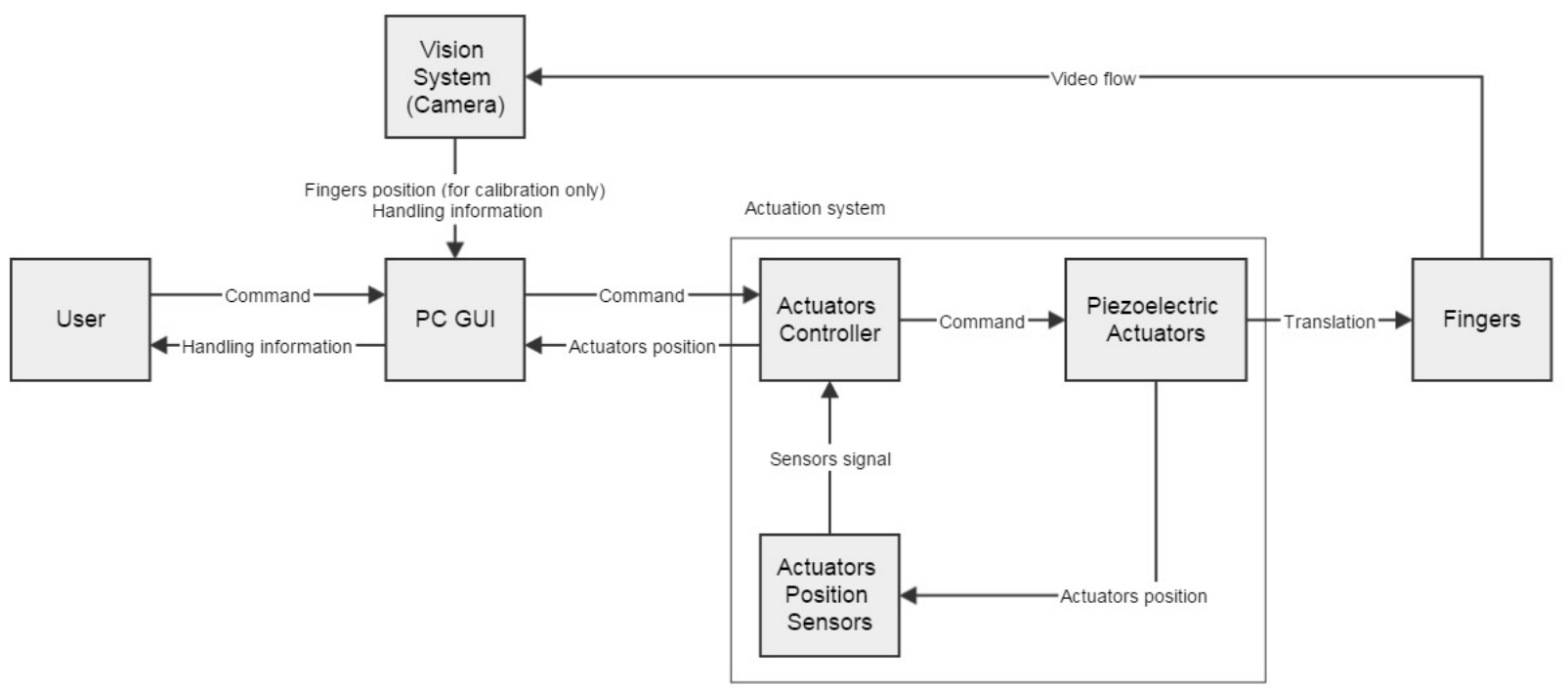

Fig. 9: Blok diagram describing the interactions between the different elements of the setup and the user. Thanks to the interface, the user can send trajectories to the actuation system. Fingers will thus be translated to execute the commanded task. A visual of micro-object manipulation is given back by the cameras.

- Perform stable in-hand regraspings:

From the stability criterion which was introduced in (8) we can deduce that:

$$
R_{d} \leq L_{\min } \mu
$$

Both criteria (8) and (17) define a minimum value for $R_{d}$. The criterion (8) is less restrictive than the (17), and only the second one (17) is thus considered.

The Modulus $E$ of the carbon fibers used in the experimental setup may vary between 200 and 600GPa, and their yield $R_{e}$ between 2 and 7GPa. We consider a medium value to define an order of magnitude of the applied forces and the internal stress: $R_{e}=4.5 G P a, E=400 \mathrm{GPa}$. Pull-off and friction values cannot be known a priori. We assume the following values of adhesion between the substrate and the

\begin{tabular}{|l|c|r|}
\hline Parameter & Notation & Value \\
\hline Minimum length of object & $L_{\min }$ & $100 \mu \mathrm{m}$ \\
Maximum length of object & $L_{\max }$ & $500 \mu \mathrm{m}$ \\
Depth of object & $\mathrm{h}$ & $100 \mu \mathrm{m}$ \\
Maximum contact surface & $S_{\text {contact }}$ & $5.10^{4} \mu \mathrm{m}^{2}$ \\
Carbon fibers yield & $R_{e}$ & $4.5 \mathrm{GPa}$ \\
Carbon fibers Young Modulus & $E$ & $400 \mathrm{GPa}$ \\
Maximum pull-off surface force & $f_{a d}^{s / o}$ & $10 \mathrm{kPa}$ \\
Minimum friction finger/object & $\mu$ & 0.25 \\
\hline
\end{tabular}

TABLE II: Parameters of the experimental setup

object, and friction between the fingers and the object: $f_{a d}^{s / o}<$ $10 k P a, \mu>0.25$. The experiments will confirm the order of magnitude of these parameters. The table II summarizes all the parameters of the experimental setup which satisfy the requirements expressed in (13) to (16). 


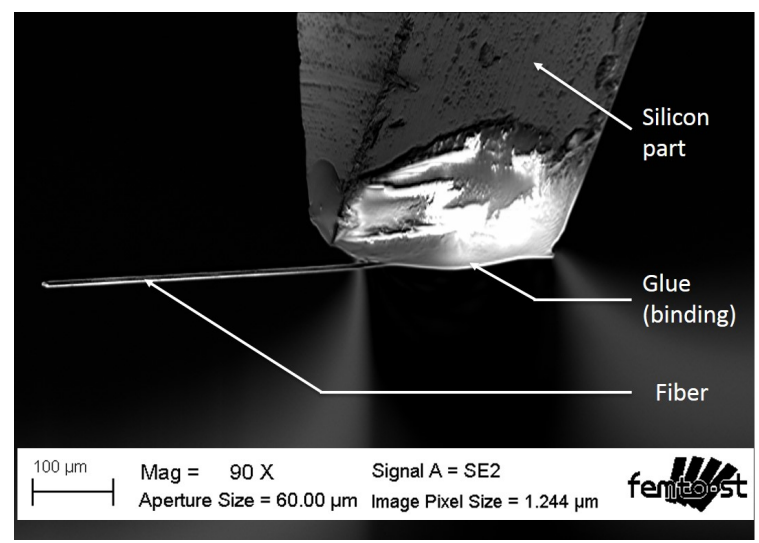

Fig. 10: Scanning Electron Microscope (SEM) photograph of a $7 \mu \mathrm{m}$ in diameter carbon fiber glued on a silicon tip.

Equation (17) provides a maximal value of $R_{d}: R_{d} \leq 8 \mu \mathrm{m}$. We choose $R_{d}=3.5 \mu \mathrm{m}$ to keep a stability margin while handling. At last, considering the chosen value of the fingers radius, the maximum fingers admissible length is $H_{d}^{\max }=$ $294 \mu \mathrm{m}$. We choose a lower value about $H_{d}=250 \mu \mathrm{m}$. The total fingers lengths are about $350 \mu \mathrm{m}$ to take into account the manipulated object thickness and the possible position uncertainties.

\section{CALIBRATION OF THE SYSTEM}

Each finger being moved independently by its own translation stages (2 DoF motion), we need to express their positions in the same frame. The front camera is used to calibrate the system thanks to the homography matrices. Each finger is moved to pre-defined positions sequence (grid like positions). The corresponding positions in the image are measured which enables computing the homography matrix between the actuators and the camera frames:

$$
\left(\begin{array}{c}
x_{i} \\
y_{i} \\
1
\end{array}\right)=H_{i}\left(\begin{array}{c}
X_{i} \\
Y_{i} \\
1
\end{array}\right)
$$

The coordinate in each actuator reference frame is simply obtained by inversing the homography matrix:

$$
\left(\begin{array}{c}
X_{i} \\
Y_{i} \\
1
\end{array}\right)=H_{i}^{-1}\left(\begin{array}{c}
x_{i} \\
y_{i} \\
1
\end{array}\right)
$$

The calibration precision will directly influence the fingers trajectory accuracy and the success of the manipulation operations.

\section{SIMULATION AND EXPERIMENTAL RESULTS}

Before performing the experimental validation of the proposed dexterous micro-manipulation method, the proposed approach was validated in simulation. Existing simulators developed for dexterous manipulation in macroscale are not fully usable in micro-manipulation. Indeed, none of the current simulators enables to simulate flexure, adhesive contact and rolling at the same time. Thus, a simulator was developed for in-hand micro-manipulation. The simulator's features and architecture is described below.

\section{Simulator description:}

- Fingers bases coordinates generation Extraction of the fingers coordinates is achieved thanks to sampling functions associated to the trajectory. The initial sampling step can be given by the user or generated automatically. An adaptive sampling is then used when the system is close to an unstable state (sliding, adhesive contact loss). All parameters, and in particular adhesion forces, can be set randomly from their nominal value, in order to validate the method robustness.

- Contact detection Contact detection enables measuring the contact position of the finger on the object. Initial contact detection is done by projecting the finger base on the object plane. Once the fingers and the manipulated object are in contact, the contact position is updated at each step. Sliding or contact loss can be known by calculating the normal and tangential components of the contact forces. Adhesion is calculated as described in the previous sections. Rolling is calculated from the object angle variation and fingers radius.

- Equilibrium position resolution

Fingers are modeled as springs that apply forces which are proportional to the deformations. The distance between the fingers base and the contact point corresponds to the spring deformation, enabling to determine the applied force $\overrightarrow{F_{e x t}}$ on the object in the $x$ and $y$ directions. The equilibrium position $\vec{P}$ is computed by inversing the stiffness matrix $K$ built from the springs stiffness:

$$
\vec{P}=-i n v(K) \overrightarrow{F_{\text {ext }}}
$$

\section{Simulation results:}

The example of the simulation of a $180^{\circ}$ rotation of a $220 \mu \mathrm{m}$ silicon square is presented in Fig.11. The fingers trajectories and forces are respectively described in Fig.11.a and Fig.11.b. This grasping sequence consists in the following steps:

- Initial approach $\left(t_{0} \rightarrow t_{1}\right)$

The three fingers come in position to begin the manipulation.

- Pick-up $\left(t_{1} \rightarrow t_{2}\right)$

Finger $2 \&$ Finger 3 perform the initial grasping without sliding thanks to a high force $F_{\text {grasping }}=450 \mu \mathrm{N}$ that ensures to overcome the pull-off force between the object and the substrate. The peak on each force curves along the $y$ axis represents the half of the simulated pull-off force.

- First rotation $\left(t_{2} \rightarrow t_{3}\right)$

The grasping forces applied by Fingers 2 and 3 are reduced to $F_{r}=225 \mu \mathrm{N}$ and a $90^{\circ}$ rotation of the object is performed. 

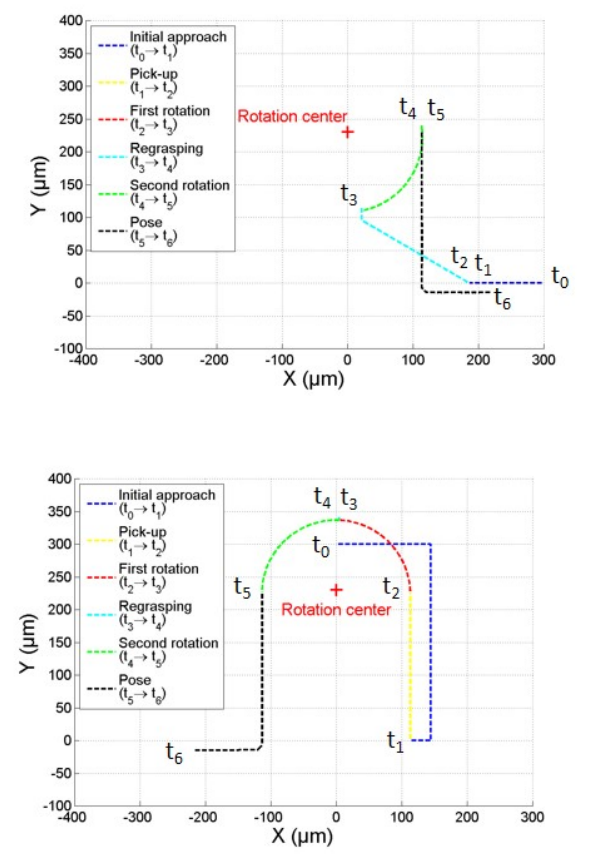

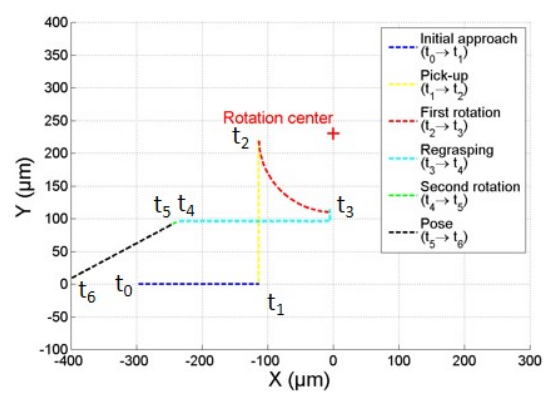

(a) : Trajectories

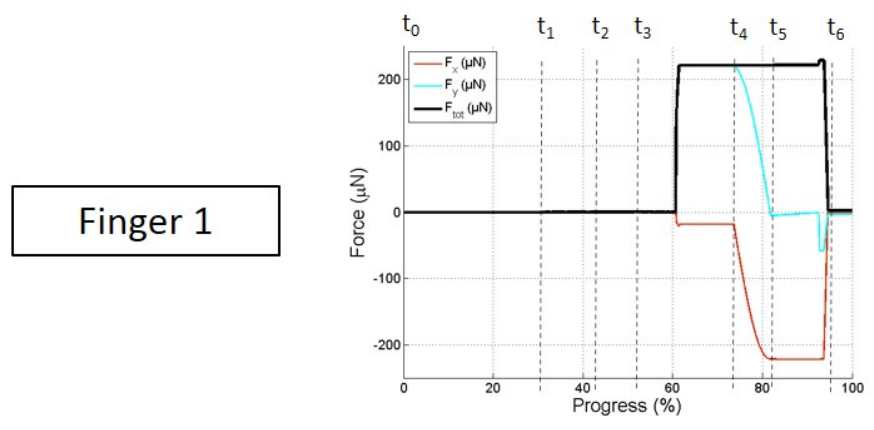

Finger 1
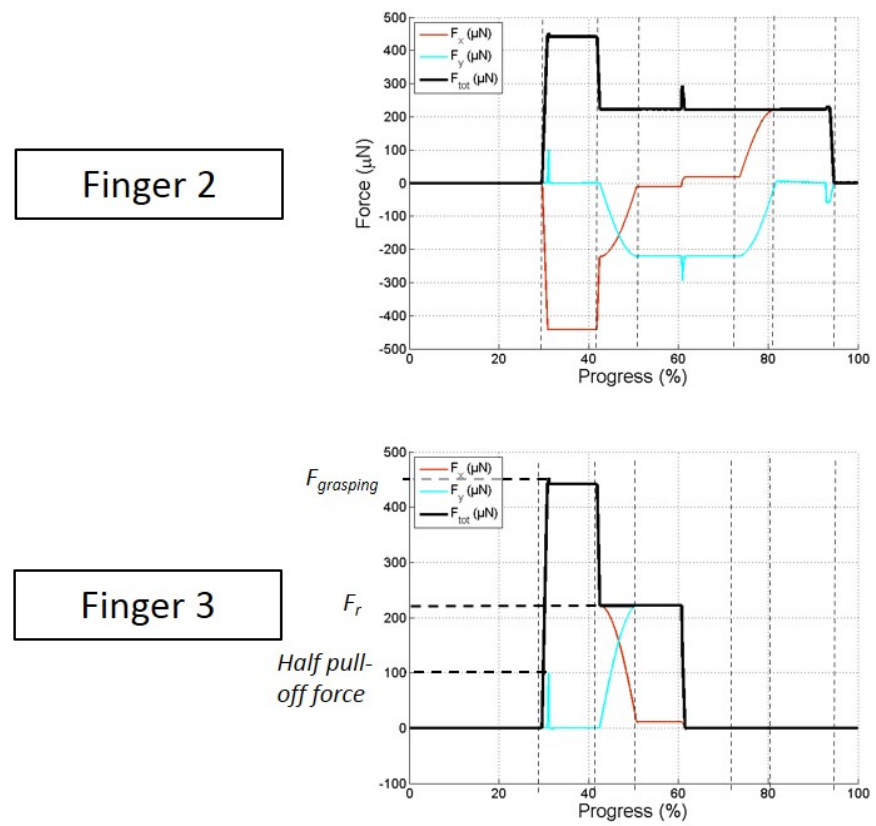

(b) : Simulated applied forces

Fig. 11: Simulation of the handling of a $220 \mu \mathrm{m}$ silicon square. Elaborated trajectories are generated (a) and then introduced in the handling simulation block that returns applied forces (b).

- Regrasping $\left(t_{3} \rightarrow t_{4}\right)$

In this step, Finger 1 takes over from Finger 3. The peak on the Finger 2 force appears when Finger 1 takes position on the object. The applied force $F_{r}$ is set to a significant lower value than $F_{\text {grasping }}$ in order to guarantee that the force is always lower than $F_{\text {grasping }}$ despite this force peak. However, the force $F_{r}$ during in-hand manipulation must be high enough to guarantee a grasping stability despite the potential adhesion disturbance that appears when Finger 3 is removed. In our experiments, $F_{r}=225 \mu \mathrm{N}$ is sufficient to change the fingers without disturbing the grasping stability.

- Rotation continuation $\left(t_{4} \rightarrow t_{5}\right)$

The second rotation is performed following the same procedure as the first one with Fingers 1 and 2.

- Object release $\left(t_{5} \rightarrow t_{6}\right)$

The object is released on the substrate while making the fingers sliding on the object edges. This explains the last peak on Fingers 1 and 2 force curves.

\section{Experimental results:}

The manipulation trajectories tested in simulation have been validated experimentally (see Fig.12). After aligning the fingers and the object thanks to the manual $z$ linear stages and calibrating the setup, the computer generates the planned trajectories to square objects, with a length of $120 \mu \mathrm{m}$ and $220 \mu \mathrm{m}$ for the silicon ones and $400 \mu \mathrm{m}$ for the SU8 ones.

The object is initially laid on a planar surface made of glass (see Fig.12.a). The fingers positions trajectories validated in simulation are executed thanks to the positioning stages controllers. No vision feedback is exploited which enables to show the robustness of the proposed method against uncertainties.

The pick-up step was successfully achieved which means that hypotheses about the frictions and the pull-off were 

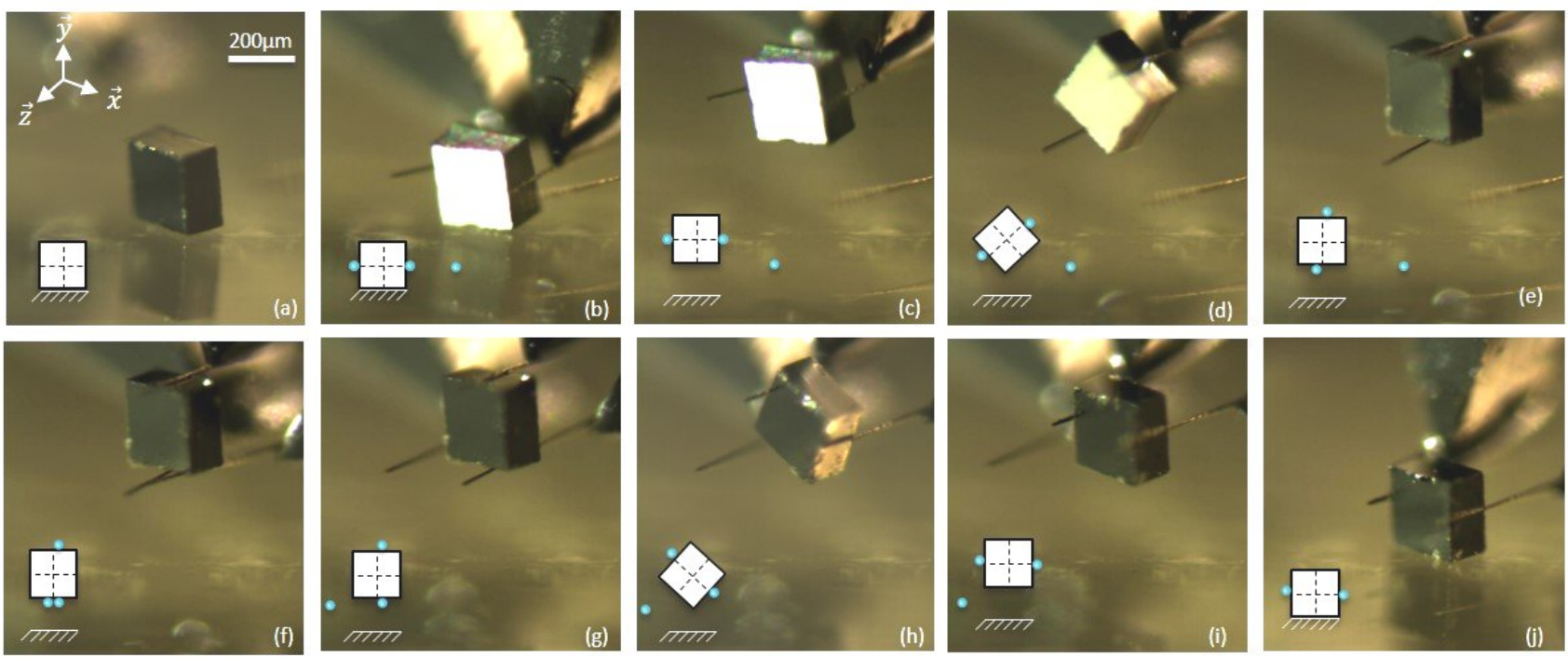

Fig. 12: Experimental in-hand manipulation of a $220 \mu \mathrm{m}$ silicon square using 3 fingers. The object is firstly detached from the substrate $(\mathrm{a} \rightarrow \mathrm{c})$. Then a first rotation is performed $(\mathrm{c} \rightarrow \mathrm{e})$. After the in-hand regrasping $(\mathrm{e} \rightarrow \mathrm{g})$, a second rotation $(\mathrm{g} \rightarrow \mathrm{i})$ enables to generate the tested $180^{\circ}$ reorientation for finally replacing the object on the substrate $(\mathrm{i} \rightarrow \mathrm{j})$.

\begin{tabular}{|c|c|c|}
\hline Finger & Axis & Manipulation range \\
\hline 1 & $\mathrm{X}$ & $277 \mu \mathrm{m}$ \\
& $\mathrm{Y}$ & $254 \mu \mathrm{m}$ \\
\hline 2 & $\mathrm{X}$ & $265 \mu \mathrm{m}$ \\
& $\mathrm{Y}$ & $355 \mu \mathrm{m}$ \\
\hline 3 & $\mathrm{X}$ & $445 \mu \mathrm{m}$ \\
& $\mathrm{Y}$ & $241 \mu \mathrm{m}$ \\
\hline Execution duration & \multicolumn{2}{|c|}{$14 \mathrm{~s}$} \\
\hline
\end{tabular}

TABLE III: Manipulation range and execution duration between the initial grasping and the pose of the object.

satisfied. A residual rotation of the object about the $z$ axis appears during the initial grasping. This is a consequence of calibration errors and fingers orientations defaults (the fingers being not exactly parallel). Positioning errors due to calibration imply that one finger comes in contact with the object before the other one. The orientation defaults of the fibers imply that the contact point is not located on the center of the object side, creating a pivot. However, we can see that the object realigns with the fibers during the manipulation. Rotations and regrasping operations were performed successfully for all object lengths. No apparent sliding has been observed during the $180^{\circ}$ object reorientation as shown on the images sequence of Fig. 12.

The stages displacement ranges and the time required in the execution of the whole manipulation sequence (Fig.12.b $\rightarrow$ Fig.12.j) are given in Table III. Note that the presented trajectories execution time was not optimized and further experiments have to be done to define the velocity limit.

\section{CONCLUSIONS AND PERSPECTIVES}

This paper has presented a new micro-handling method that exploits dexterity provided by a 3-compliant beams manipulation system. A detailed study has been performed to manipulate micro-objects having parallel edges. Manipulation procedure includes the grasping step, the in-hand rotation and the finger gaiting. The stability of each step has been studied considering the worst case. The trajectory planning associated to the defined steps and the fingers design methods have been also provided. Automatic translations and rotations over $180^{\circ}$ degrees of objects with sizes down to $120 \mu \mathrm{m}$ have successfully been performed. This demonstration shows that this approach has a high potential in micro-assembly.

Based on the proof of concept presented in this paper, an automatic 3D handling methods associated to an enhanced setup will be developed. More complex object shapes will be handled thanks to the trajectory planner developed in [15]. Furthermore, rotations about additional axes will be studied enabling out of the plane rotations. Integrated dexterous

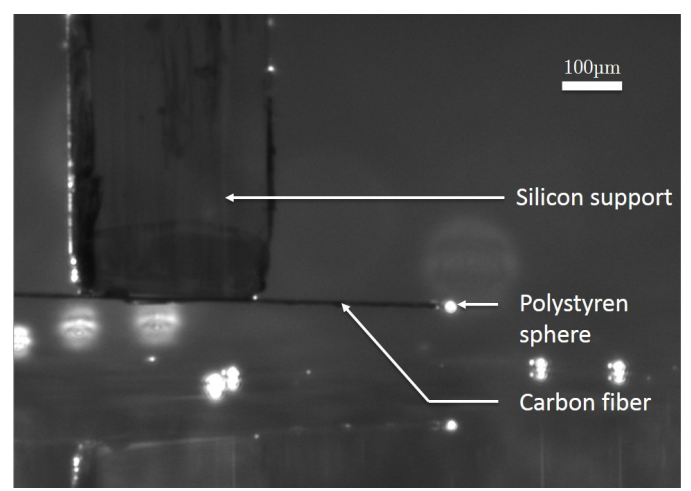

Fig. 13: $20.3 \mu \mathrm{m}$ in diameter polystyren sphere sticked on a fiber tip. The punctual contact between the micro-sphere and the manipulated object would allow 3D dexterous micromanipulation. 
piezoelectric microgrippers will be used to perform assembly tasks. More DoF will be integrated to the manipulation system and a fourth finger will be added. To guarantee punctual contact between the fingers and the manipulated object, micro-spheres will be fixed to the fingers tips and used as end-effectors as shown in Fig.13. This will result in better estimation of the contact point's positions and enables 3D motion control of the manipulated micro-object. Another ambitious perspective is to perform dexterous manipulation at the nanoscale.

\section{ACKNOWLEDGMENT}

This work has been supported by: Labex ACTION project (contract "ANR-11-LABX-0001-01"), Equipex ROBOTEX project (contract "ANR-10-EQPX-44-01"), French RENATECH network and its FEMTO-ST technological facility, Region of Franche-Comté, Percipio Robotics SA Company.

\section{REFERENCES}

[1] M. Savia and H. Koivo, "Contact micromanipulation-Survey of strategies," Mechatronics, IEEE/ASME Transactions, vol. 14, no. 4, pp. 504-514, 2009.

[2] C. Clevy, A. Hubert, and N. Chaillet, "Micromanipulation and microassembly systems," International Journal of Advanced Robotic Science, 2006.

[3] B. Tamadazte, E. Marchand, S. Dembele, and N. Le Fort-Piat, "CAD Model-based Tracking and 3D Visual-based Control for MEMS Microassembly," The International Journal of Robotics Research, vol. 29, no. 11, pp. 1416-1434, 2010.

[4] F. Arai, T. Fukuda, and K. Iwata, H. andItoigawa, Fifth International Conference on Advanced Robotics 'Robots in Unstructured Environments, 1991.

[5] Y.-S. Kim, J.-M. Yoo, S. H. Yang, Y.-M. Choi, N. G. Dagalakis, and S. K. Gupta, "Design, fabrication and testing of a serial kinematic MEMS XY stage formultifinger manipulation," Journal of Micromechanics and Microengineering, vol. 22, 2012.

[6] H. K. Chu, J. K. Mills, and W. L. Cleghorn, "Dual-arm micromanipulation and handling of objects through visual images," in Proc. Int Mechatronics and Automation (ICMA) Conf, 2012, pp. 813-818.

[7] Y. Matsuzaki, K. Inoue, and S. Lee, "Manipulation of micro-scale objects using micro hand with two rotational fingers," in Proc. Int. Symp. Micro-NanoMechatronics and Human Science MHS 2009, 2009, pp. 438-443.

[8] H. Xie and S. Régnier, "Development of a flexible robotic system for multiscale applications of micro/nanoscale manipulation and assembly," IEEE/ASME Transactions on Mechatronics, vol. 16, no. 2, pp. 266-276, 2011.

[9] J. D. Wason, J. T. Wen, and N. G. Dagalakis, "Dextrous manipulation of a micropart with multiple compliant probes through visual force feedback," in 2011 IEEE International Conference on Robotics and Automation. Ieee, May 2011, pp. 5991-5996.

[10] S. Krishnan and L. Saggere, "A multi-fingered micromechanism for coordinated micro/nano manipulation," Journal of Micromechanics and Microengineering, vol. 17, no. 3, pp. 576-585, Mar. 2007.

[11] Q. Zhou, P. Korhonen, J. Laitinen, and S. Sjovall, "Automatic dextrous microhandling based on a 6 dof microgripper," Journal of Micromechatronics, vol. 3, no. 3-4, pp. 359-387, 2006.

[12] E. Shimada, J. Thompson, J. Yan, R. Wood, and R. Fearing, "Prototyping millirobots using dextrous microassembly and foldings," in ASME International Mechanical Engineering Congress and Exposition, 2000.

[13] J. Thompson and R. Fearing, "Automating Microassembly with Orthotweezers and Force Sensing," in IEEE/RSJ International Conference on Intelligent Robots and Systems, 2001.

[14] R. Fearing and E. Shimada, "Apparatus and method for manipulation of an object," Patent US6798 120 B1, 09 28, 2004.

[15] J.-A. Seon, R. Dahmouche, and M. Gauthier, "Trajectory planner for dexterous in-hand micromanipulation taking into account adhesion forces," in IEEE/RSJ International Conference on Intelligent Robots and Systems (submitted), 2015. 